Ann. Zootech., I979, 28 (2), 145-I57.

I. N.R.A.
BIbliotheque UO 3590.
DOMAINe DE CROUELLE
63039
CLERMONT-FD CEDEX?

\title{
Influence de l'agglomération et du stockage des aliments composés sur leur teneur en Canthaxanthine : conséquences sur la digestibilité et la fixation de ce pigment chez la Truite Arc-en-ciel
}

\author{
G. CHOUBERT (Jr) et P. LUQUET \\ avec la collaboration technique de R. Cescosse, Y. Hontang et R. LANnkBere, \\ Laboratoire de Nutrition des Poissons \\ Centre de Recherches Hydrobiologiques, I.N.R.A., \\ St-Pée-sur-Nivelle, 64310 Ascain (France)
}

\section{Résumé}

Afin de déterminer une des causes de variabilité de la pigmentation des truites en pisciculture, les pertes en canthaxanthine de I 3 échantillons de "CAROPHYL $I_{+}$rouge " et de 5 échantillons de "Canthaxanthine ro p. Ioo hydrosoluble " ont été examinées au cours de la conservation des concentrats, des processus d'agglomération et de stockage des aliments avant distribution. Les conséquences de cette dégradation sur la digestibilité et la fixation de ce pigment par la truite arc-en-ciel ont été ensuite étudiées.

La protection de la canthaxanthine dans les concentrats est efficace et les différents lots de fabrication homogènes. Les teneurs en produit pur sont stables et correspondent aux valeurs théoriques.

Bien que la température des granulés à la sortie de la presse soit modérée $\left(43^{\circ} \mathrm{C}\right)$ le processus de granulation entraine la perte d'une fraction importante de pigments. Cette diminution est significative et égale à $20 \mathrm{p}$. Ioo et $\mathrm{s} 5 \mathrm{p}$. I oo repsectivement pour les modes de présentation "CAROPHYLL rouge " et "Canthaxanthine $10 \mathrm{p}$ roo hydrosoluble ".

Au cours d'un stockage des granulés, d'une durée de 2 mois, à la température hivernale ambiante et avant utilisation on a noté une perte supplénentaire significative d'environ $\mathrm{i} 7 \mathrm{p}$. 100 quel que soit le mode de présentation.

Les tests in vivo indiquent une faible digestibilité de la canthaxanthine (20 p. 10o). Ces valeurs basses peuvent s'expliquer, partiellement du moins, par la méthode utilisée pour la récolte des fèces.

Bien que le rapport de la canthaxanthine fixée à la canthaxanthine ingérée soit voisin de I p. Ioo la couleur de la chair des truites est fortement prononcée quelle que soit la préparation utilisée.

\section{Introduction}

La pigmentation de la peau et de la chair des salmonidés est due aux pigments caroténoïdes contenus dans leurs tissus. En milieu naturel, la couleur rouge des tissus des truites et des saumons provient de leur nourriture (LEDERER, I935; 
Goodwin, I952), crustacés notamment dont le pigment dominant, l'astaxanthine (KUHN et SORENSEN, I938) est accumulé dans les tissus sans transformation d'aucune sorte (STEven, I948; Hata et Hata, I973).

En salmoniculture intensive, la pigmentation des truites est obtentue soit par complémentation de l'aliment par des déchets industriels de crustacés (LAMBERTSEN et BRAEKKAN, I97I) soit par supplémentation des régimes alimentaires avec de la canthaxanthine, pigment caroténoïde synthétisé industriellement (ISLER, OFNER et SIEMERS, I958).

Dans les conditions pratiques de pisciculture, les résultats obtenus pour la pigmentation des salmonidés sont très variables tant entre lots qu'intra lot.

Si quelques facteurs liés au poisson et au milieu ont été décrits, notamiment les facteurs génétiques (BESSE, I95I; GoODwIN, I952), les facteurs physiologiques (Steven, I949; ShNarevich et Sakhnenko, I97I), les facteurs pathologiques dont le rôle a été mis en évidence chez le poulet de chair (BIRD, I953; Yvore et MAInguy, I972) et les paramètres du milieu (Sumner et Fox, I933; Peterson et al., I966); il n'en est pas de même des facteurs liés à l'aliment. Seuls quelques effets des apports, tels que la nature et la quantité des pigments caroténoïdes, ou les effets des autres éléments de la ration alimentaire ont été déjà partiellement étudiés (ABDUL-MALAK, I975; ABdul-MaLAK et al., I975; ChOUBERT et LuQUE'T, I975; ChOUBERT, I977).

L'instabilité des pigments caroténoïdes pose le problème de leur conservation dans les matières premières et les aliments fabriqués. Les conditions et la durée de conservation peuvent apporter les modifications sensibles difficilement prévisibles étant donné la variété des formules alimentaires, les conditions de fabrication et de distribution dans la pratique industrielle. (MaINGUY et Rougues, I965). Ces remarques ne sont pas limitées aux seuls pigments caroténoïdes mais s'étendent aux autres constituants de la ration alimentaire tels que les lipides, les protéines ou les vitamines. Ainsi, les pertes en vitamines $\mathrm{C}$ atteignent 25 à $50 \mathrm{p}$. I 00 par suite de l'agglomération (WORNICK, I960) et 97 p. Ioo après extrusion (SLINGER, RAZzAQUE et CHo, I978). Après quelques semaines de stockage dans des conditions normales de température, les pertes constatées sont de l'ordre de 25 à 30 p. IOo (Wornick, I960). Pour la vitamine A, les pertes observées lors du stockage sont du même ordre de grandeur (20 p. IOO) (VALDEBOUZE et LEVY, I972).

Pour tenter d'expliquer les différences dans la pigmentation des truites d'élevage, il nous a paru utile d'examiner d'une part la variabilité entre les différents lots de fabrication de concentrat de canthaxanthine et les conséquences d'un stockage avant incorporation, d'autre part les pertes inhérentes aux processus de fabrication et de stockage de l'aliment. L'influence de ces différents paramètres sur la digestibilité et la fixation de la canthaxanthine par la truite arc-en-ciel. a ensuite été examinée.

\section{Matériels et méthodes}

\section{I. - Concentrats de canthaxanthine}

Deux préparations de canthaxanthine sont étudiées :

- une préparation dénommée "CAROphyl, rouge " $\left(^{*}\right)$ : I3 échantillons,

- une préparation dénommée "Canthaxanthine ro p. Ioo hydrosoluble " $\left(^{*}\right)$ : 5 échantillons.

(*) Société F. Hofmann, La Roche, 52, boulevard du Parc, 9252 I Neuilly-sur-Seine. 
Ces deux formes sont celles des préparations commerciales habituelles pour l'alimentation car ces concentrats proviennent d'une part de sept fabricants d'aliment pour poisson représentant plus des trois quart de la production française recensée et d'autre part du fabricant lui-même. Dix huit échantillons représentant I l lots de fabrication sont ainsi analysés.

\section{2.- Aliment}

Chacune de ces préparations est introduite dans un aliment de type commercial ne contenant pas de caroténoïdes, de façon à réaliser une concentration d'environ $220 \mathrm{ppm}$ de produit pur, concentration recommandée par le fabricant. Cet aliment contient de plus, environ $0,5 \mathrm{p}$. Ioo d'oxyde de chrome pour les études de digestibilité. L'aliment est granulé par une presse de laboratoire CPM sans adjonction de vapeur (diamètre des perforations de la filière $=2,5 \mathrm{~mm}$ ). Afin de faciliter le passage de la farine à travers la filière, de l'eau (environ $2 \mathrm{p}$. roo du mélange) est ajoutée. I a température à la sortie de la presse est notée au cours de chaque fabrication. Les aliments sont conservés sous abri dans des sacs en papier ( 5 couches), le stockage, avant utilisation, a lieu dans un bâtiment non fermé à proximité des bassins d'élevage et à la température ambiante (température minimum $6, \mathrm{I}{ }^{\circ} \mathrm{C}$, température maximum I $6,6^{\circ} \mathrm{C}$.).

La teneur en canthaxanthine est déterminée sur les concentrats, l'aliment en farine, 1'aliment granulé à la sortie de la presse et après deux mois de stockage.

\section{3. - Poissons}

L'expérience d'une durée de 36 jours s'est déroulée à la pisciculture expérimentale de Donzacq (Landes). I 8 lots de 80 truites arc-en-ciel (Salmo gairdnerii Rich.) d'un poids initial de r $30 \mathrm{~g}$ sont constitués au hasard puis placés dans des bassins rectangulaires de $2 \mathrm{~m}^{2}$ de surface. La hauteur d'eau est d'environ $40 \mathrm{~cm}$. La température de l'eau provenant de sources est pratiquement constante toute l'année $\left(\mathrm{I} 7{ }^{\circ} \mathrm{C}\right)$. Les truites sont nourries à volonté par repas, 4 fois par jour.

En fin d'expérience, un échantillon de ro truites est prélevé pour analyse. Celles-ci sont tuées par rupture des vertèbres cervicales puis pesées. La musculature épaxiale d'une part et le reste d'autre part sont prélevés. Les échantillons provenant des différents poissons sont congelés, regroupés et conservés au froid $\left(-\mathrm{I} 8{ }^{\circ} \mathrm{C}\right)$ jusqu'au broyage qui précède immédiatement les analyses. Les autres truites sont maintenues dans les mêmes conditions pour l'étude de la digestibilité par la méthode indirecte qui consiste à mesurer la concentration de la canthaxanthine par rapport à celle d'un marqueur inerte : l'oxyde de chrome (E,DIN, I9I8).

Les fèces sont prélevées, après anesthésie des poissons, par pression abdominale (Singh et Nose, I967) à 9 heures et I7 heures (Possompes, I973). Les fèces sont alors congelées, groupées et 1yophilisées.

\section{4. - Déterminations analytiques}

La couleur peut être caractérisée par trois critères : la teinte, la saturation et l'éclat. L'éclat étant difficile à percevoir dans le cas de la chair, nous n'avons retenu que la teinte et la saturation. L'appréciation de ces deux critères s'effectue 
visuellement, sur le terrain, par comparaison avec la charte Kodak Color séparation guide NQ I3, puis est confirmée en laboratoire sur des photographies en couleur comportant sur le cliché l'image de la chair des truites ainsi que celle de cette même charte.

Le dosage de la canthaxanthine est effectué en double, selon la méthode décrite par OSADCA, Araujo et de RITTER (I972) sur $20 \mathrm{~g}$ de tissus frais, I5 g d'aliment sec et $\mathrm{I} g$ de fèces préalablement lyophilisées.

L'oxyde de chrome est dosé selon la méthode de Borin, King et Ki,ostermax (1952) sur $500 \mathrm{mg}$ d'aliment frais, et roo $\mathrm{mg}$ de fèces lyophilisées.

\section{Résultats}

\section{I. - Concentrat en canthaxanthine}

Les résultats concernant les teneurs en canthaxanthine des différents concentrats sont groupés dans le tableau I. On note que les teneurs en canthaxanthine sont homogènes pour l'ensemble des lots analysés tant pour la préparation "CAROPHYL, rouge " (moyenne $10,5 \pm 0,5$ ), que pour la préparation "Canthaxanthine Io p. Ioo hydrosoluble " (moyenne Io,o $\neq 0,6$ ).

Il n'apparaît donc, aucune différence entre les teneurs en canthaxanthine des échantillons de provenances diverses.

\section{2. - Stabilité de la canthaxanthine}

Les résultats de dosage de la canthaxanthine dans l'aliment en farine, l'aliment aggloméré et l'aliment aggloméré stocké pendant 2 mois sont rapportés dans le tableau 1 .

On constate tout d'abord que le taux de canthaxanthine de la farine varie de 240 à $310 \mu \mathrm{g} / \mathrm{g}$. Ces taux sont, en moyenne, supérieurs aux taux théoriques 26 p. roo et 20 p. roo respectivement pour les préparations de canthaxanthine "CARophyli, rouge " et "Canthaxanthine ro p. roo hydrosoluble".

\section{a. - Infuence de l'agglomération}

A la sortie de la presse, la température des granulés oscille entre 42 et $43{ }^{\circ} \mathrm{C}$. La teneur en canthaxanthine accuse un fléchissement général (sauf pour un échantillon) après la granulation. Pour la préparation "CAROPHYL. rouge " cette perte d'environ 20 p. 100 est significative, de même que la perte notée pour la préparation "Canthaxanthine Io p. Ioo hydrosoluble ", bien qu'inférieure (de l'ordre de I5 p. Ioo).

Cependant, le calcul statistique, établi en comparant les moyennes, ne fait pas apparaître de différence significative entre la stabilité vis-à-vis de l'influence de la presse des formes "CAROPHYLI rouge " et "Canthaxanthine Io p. Ioo hydrosoluble ».

\section{b. - Influence du stockage}

Les teneurs en canthaxanthine des aliments agglomérés conservés en sac papier pendant deux mois, à température ambiante, diminuent significativement 
TABLEAU I

Concentration en canthaxanthine, rapportée à la matiève sèche, des concentrats, de l'aliment avant et après granulation et de l'aliment stocké pendant deux mois

Canthaxanthin concentration (relative to dry matter) of feed concentrate, feed before and after pelleting and two months old pellets

\begin{tabular}{|c|c|c|c|c|c|c|}
\hline $\begin{array}{c}\mathrm{N}^{\circ} \text { de } \\
\text { fabrication } \\
\text { Lot sumber }\end{array}$ & $\begin{array}{l}\text { Fournisseur } \\
\text { Supplier }\end{array}$ & $\begin{array}{c}\text { No de } \\
\text { l'aliment } \\
\text { Ficed number }\end{array}$ & $\begin{array}{c}\text { Concentrat } \\
\text { Concentrate } \\
\text { (p. Ioo) }\end{array}$ & $\begin{array}{c}\text { Aliment } \\
\text { avant } \\
\text { granulation } \\
\text { Meal } \\
(\mu \mathrm{g} / \mathrm{g})\end{array}$ & $\begin{array}{c}\text { Aliment } \\
\text { après } \\
\text { granulation } \\
\text { Pellet } \\
(\text { ug } / \mathrm{g})\end{array}$ & $\begin{array}{l}\text { Aliment } \\
\text { stock } \\
\text { pendant } \\
2 \text { mois } \\
2 \text { month } \\
\text { old pellets } \\
(\text { ug } / \mathrm{g})\end{array}$ \\
\hline \multicolumn{7}{|c|}{ CAROPIIYLI, rouge $\left(C_{\text {cirophyll }} R c d\right)$} \\
\hline $3129 .$. & $\begin{array}{l}A \\
\mathrm{~J} \\
\mathrm{C}\end{array}$ & $\begin{array}{l}\text { I } \\
2 \\
3\end{array}$ & $\begin{array}{r}9,9 \\
11,0 \\
10,5\end{array}$ & $\begin{array}{l}249 \\
282 \\
276\end{array}$ & $\begin{array}{l}218 \\
289 \\
198\end{array}$ & $\begin{array}{l}142 \\
170 \\
205\end{array}$ \\
\hline $274^{\mathrm{I}}$ & $\begin{array}{l}\text { A } \\
\text { I } \\
\text { J }\end{array}$ & $\begin{array}{l}4 \\
5 \\
6\end{array}$ & $\begin{array}{l}10,5 \\
10,3 \\
\text { 10,9 }\end{array}$ & $\begin{array}{l}264 \\
262 \\
324\end{array}$ & $\begin{array}{l}242 \\
221 \\
223\end{array}$ & $\begin{array}{l}165 \\
201 \\
211\end{array}$ \\
\hline 3105 & $\begin{array}{l}C \\
\mathrm{~J}\end{array}$ & $\begin{array}{l}7 \\
8\end{array}$ & $\begin{array}{l}\text { I0,6 } \\
\text { г } 0,7\end{array}$ & $\begin{array}{l}240 \\
318\end{array}$ & $\begin{array}{l}173 \\
267\end{array}$ & $\begin{array}{l}171 \\
218\end{array}$ \\
\hline 2600 & $E$ & 9 & I 0,6 & 234 & 216 & I 73 \\
\hline I 609 & G & Io & 9,5 & 306 & $2 \mathrm{I}_{2}$ & I 76 \\
\hline 997 & $\mathrm{H}$ & I I & 9,7 & 317 & $2 \mathrm{I} 2$ & I 80 \\
\hline $\begin{array}{l}\text { Inconnu } \\
\text { Unknown }\end{array}$ & D & 12 & 10,2 & 240 & I 88 & I 54 \\
\hline $\begin{array}{l}\text { Inconnu. . } \\
\text { Unknozen }\end{array}$ & F & I 3 & 10,8 & 299 & 216 & 178 \\
\hline \multicolumn{3}{|c|}{ Moyenne (Mean) } & $\begin{array}{r}10,5 \\
\therefore \quad 0,5\end{array}$ & $\begin{array}{r}277 \\
\therefore \quad 32\end{array}$ & $\begin{array}{r}221 \\
-\quad 32\end{array}$ & $\begin{array}{r}180 \\
\quad \quad 22\end{array}$ \\
\hline
\end{tabular}

Canthaxanthine Io p. Ioo hydrosoluble (Io p. Ioo hydrosoluble canthaxanthin)

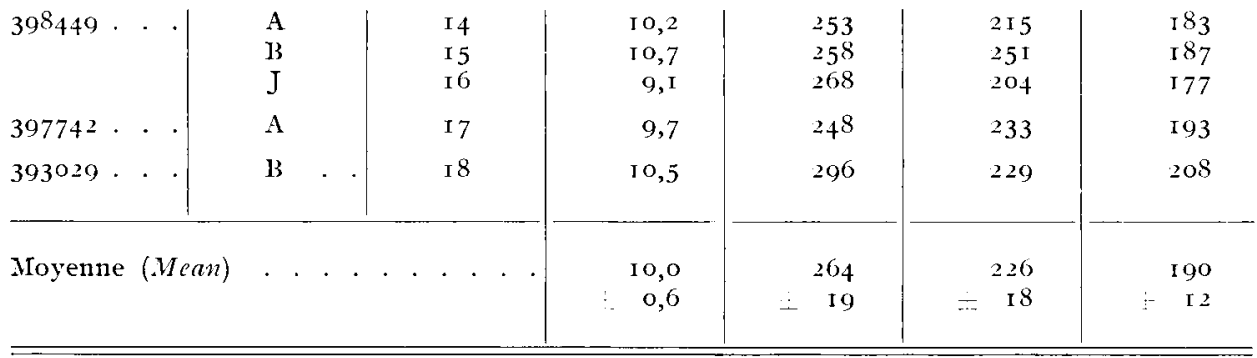

de façon semblable pour les deux préparations. Cette perte atteint I 8 p. roo pour la préparation "CAROPHYL, rouge" et 16 p. Ioo pour la préparation "Canthaxanthine ro p. roo hydrosoluble $\%$. 
Aucune différence significative n'apparaît entre les pertes observées au cours du stockage pour les deux modes de préparation.

\section{3. - Pigmentation des truites}

\section{a. - Appréciation visuelle}

Dans la présente expérience, toutes les préparations de canthaxanthine modifient la couleur du muscle des truites en déplaçant la teinte vers l'orangé.

\section{TABIEAU 2}

Concentration en canthaxanthine du muscle des truites et du poisson entier

Rapport quantité de canthaxanthine fixée par le poisson/quantité de canthaxanthine ingivie

Canthaxanthin concentration in trouts and trout muscle

Ratio of canthaxanthin fixed to canthaxanthin ingested by the fish

\begin{tabular}{|c|c|c|c|}
\hline $\begin{array}{c}\text { Lot de poisson } \\
\text { correspondant } \\
\text { aux échantillons } \\
\text { de canthaxanthine } \\
\text { Fish group } \\
\text { corresponding to } \\
\text { canthaxanthin samples } \\
(\text { Table I) }\end{array}$ & $\begin{array}{l}\text { Concentration } \\
\text { en canthaxanthine } \\
\text { du muscle } \\
\text { (ug ig poids sec) } \\
\text { Muscle } \\
\text { canthaxanthin } \\
\text { conccutration } \\
\text { (ug } / g \text { dry matter) }\end{array}$ & $\begin{array}{l}\text { Concentration } \\
\text { en canthaxanthine } \\
\text { du poisson entier } \\
\text { (ㅆg poicis sec) } \\
\text { Whole Fish } \\
\text { canthaxanthin } \\
\text { concentration } \\
\text { (ug dry matter) }\end{array}$ & $\begin{array}{c}\text { Rapport } \\
\text { Quantité fixée } \\
\text { Quantité ingérée } \\
\text { Fixed } \\
\text { Quantities ingested } \\
\text { Quantitics ratio }\end{array}$ \\
\hline \multicolumn{4}{|c|}{ CAROPHYLL rouge (Carophyll Red) } \\
\hline $\begin{array}{l}1 \\
2 \\
3 \\
4 \\
5 \\
6 \\
7 \\
8 \\
9 \\
10 \\
11 \\
12 \\
13\end{array}$ & $\begin{array}{l}3 \mathrm{I}, 99 \\
57,00 \\
27,23 \\
50,24 \\
47,79 \\
5 \mathrm{I}, 53 \\
27,09 \\
40,42 \\
15,86 \\
13,21 \\
3 \mathrm{I}, 34 \\
33,00 \\
50,75\end{array}$ & $\begin{array}{l}0,86 \\
1,83 \\
1,16 \\
1,99 \\
1,59 \\
1,56 \\
0,99 \\
1,36 \\
0,76 \\
0,69 \\
0,83 \\
1,24 \\
1,63\end{array}$ & $\begin{array}{l}0,65 \\
1,07 \\
0,92 \\
1,28 \\
1,23 \\
1,13 \\
0,93 \\
0,82 \\
0,64 \\
0,49 \\
0,63 \\
1,07 \\
1,21\end{array}$ \\
\hline Moyenne $($ Mean $)$. . & $\begin{array}{r}36,73 \\
-\quad 14,08\end{array}$ & $\begin{array}{r}1,27 \\
+\quad 0,43\end{array}$ & $\begin{array}{r}0,93 \\
: \quad 0,26\end{array}$ \\
\hline
\end{tabular}

Canthaxanthine Io p. Ioo hydrosoluble (Io p. Ioo hydrosoluble canthaxamhin)

\begin{tabular}{|c|c|c|c|}
\hline $\begin{array}{l}\text { I } 4 \\
\text { I } 5 \\
\text { I } 6 \\
\text { I } 7 \\
\text { I } 8\end{array}$ & $\begin{array}{l}36,67 \\
39,02 \\
28,75 \\
20,88 \\
23,63\end{array}$ & $\begin{array}{l}:, 23 \\
1,39 \\
1,73 \\
0,63 \\
0,72\end{array}$ & $\begin{array}{l}0,86 \\
0,85 \\
\mathrm{I}, 23 \\
0,41 \\
0,53\end{array}$ \\
\hline Moyenne $($ Mean $)$. & $\begin{array}{r}30,99 \\
\therefore \quad 7,17\end{array}$ & $\begin{array}{r}1,14 \\
+\quad 0,46\end{array}$ & $\begin{array}{r}0,77 \\
+\quad 0,32\end{array}$ \\
\hline
\end{tabular}


On peut noter une saturation supérieure de la teinte du muscle des truites recevant les préparations de canthaxanthine " $\mathrm{CAROPHYL}_{\mathrm{L}}$ rouge " et une saturation moindre pour celles recevant les préparations "Canthaxanthine io p. Ioo hydrosoluble". Cette différence, toutefois, ne peut être quantifiée.

\section{b. - Appréciation chimique}

Les concentrations en canthaxanthine du muscle et des truites entières sont rapportées dans le tableau 2 .

On note que le caractère essentiel des résultats obtenus est un cofficient de variations toujours très élevé.

Les moyennes des concentrations en canthaxanthine du muscle sont de $37 \mu \mathrm{g} / \mathrm{g}$ en moyenne pour les truites recevant la préparation "CAROPHYLI rouge " et de $3 \mathrm{I} \mu \mathrm{g} / \mathrm{g}$ en moyenne pour celles recevant la préparation "Canthaxanthine Io $p$. I oo hydrosoluble "; ces concentrations varient de I à 4 pour les truites recevant la préparation "CAROPHYL, rouge " ( $\mathrm{r}_{3}, 2$ à $57 \mu \mathrm{g} / \mathrm{g}$ ) et de I à 2 pour les truites recevant la préparation "Canthaxanthine Io p. Ioo hydrosoluble " $(20,88$ à 39,02 $\mu \mathrm{g} / \mathrm{g})$.

Aucune différence significative n'est à mentionner entre les concentrations en pigments de $I, 27 \mu \mathrm{g} / \mathrm{g}(0,69$ à $\mathrm{I}, 99 \mu \mathrm{g} / \mathrm{g})$ pour les truites recevant les préparations "CAROPHYLL rouge " et de I, I $4 \mu \mathrm{g} / \mathrm{g}(0,63$ à $\mathrm{I}, 73 \mu \mathrm{g} / \mathrm{g})$ pour celles recevant les préparations "Canthaxanthine Io p. Ioo hydrosoluble ". Ces valeurs moyennes ne diffèrent pas significativement entre elles.

Chez les poissons recevant les préparations "CAROPHyLI, rouge " on remarque une forte corrélation entre la teneur en canthaxanthine du muscle et celle du poisson tout entier. On ne retrouve pas semblable liaison chez l'animal recevant les préparations "Canthaxanthine ro p. roo hydrosoluble ".

\section{c. - Rapports canthaxanthine fixée/canthaxanthine ingérée (tabl. 2)}

Afin de calculer le rapport canthaxanthine fixée/canthaxanthine ingérée nous avons considéré les concentrations en canthaxanthine trouvées dans les granulés juste après granulation. Le rapport canthaxanthine fixée/canthaxanthine ingérée est en moyenne égal à 0,93 pour les préparations "CAROPHYLLr ouge " et 0,77 pour les préparations "Canthaxanthine Io p. I oo hydrosoluble ". I1 ne ressort aucune différence significative entre ces deux types de préparation. Pourtant on note également une certaine dispersion des résultats : I à 3 tant pour les préparations "CAROPHYLL rouge " $(0,49-\mathrm{I}, 28)$ que pour les préparations "Canthaxanthine Io $\mathrm{p}$. Ioo hydrosoluble " $(0,4 \mathrm{I}$ à $I, 23)$.

\section{4. - Utilisation digestive apparente de la canthaxanthine}

Les cœfficients d'utilisation digestive apparente (C.U.D.) de la canthaxanthine sont rapportés dans le tableau 3. On remarque une très grande dispersion des résultats. Ceux-ci peuvent varier de I à ro $(4,8$ à 49,4 p. Ioo) pourlespréparations "CARophyi.I rouge " et de I à $2(20,9$ à 43,2 p. IOo) pour les préparations "Canthaxanthine Io p. Ioo hydrosoluble ».

Aucun effet significatif de la préparation ne peut être mis en évidence sur l'utilisation digestive de la canthaxanthine par la truite bien que les valeurs moyennes soient supérieures pour les préparations "Canthaxanthine ro p. 100 hydrosoluble " à celle des préparations "CAROPHYL $I_{\text {I }}$ rouge ". 
TABLEAU 3

Cafficient d'utilisation digestive appavente de la canthaxanthine. Rapport quantité de canthaxanthine fixée par le poisson /quantité de canthaxanthine absorbée

Apparent digestibility cofficient of canthaxanthin. Ratio of canthaxanthin fixed to canthaxanthin absorbed by the fish

\begin{tabular}{|c|c|c|}
\hline $\begin{array}{l}\text { Lot de poisson } \\
\text { correspondant } \\
\text { aux échantillons } \\
\text { de canthaxanthine } \\
\text { Fish group corresponding } \\
\text { to canthaxanthin samples } \\
(\text { Table I) }\end{array}$ & $\begin{array}{c}\text { Cofficient d'utilisation } \\
\text { digestive apparente } \\
\text { de la canthaxanthine } \\
\text { Apparent digestibility } \\
\text { cofficient of canthaxanthin } \\
\text { (p. Ioo) }\end{array}$ & $\begin{array}{l}\text { Rapport Quantité de } \\
\text { canthaxanthine fixée par } \\
\text { le poisson/Quantité de } \\
\text { canthaxanthine absorbée } \\
\text { Fixed canthaxanthin } \\
\text { Absorbed canthaxanthin } \\
\text { ratio }\end{array}$ \\
\hline \multicolumn{3}{|c|}{ CAROPHYLL rouge (Carophyll Red) } \\
\hline $\begin{array}{r}\text { I } \\
2 \\
3 \\
4 \\
5 \\
6 \\
7 \\
8 \\
9 \\
\text { I0 } \\
\text { I I } \\
\text { I } 2 \\
\text { I } 3\end{array}$ & $\begin{array}{r}7,8 \\
4 \mathrm{I}, 4 \\
4,8 \\
34,1 \\
27,2 \\
14,6 \\
\mathrm{I} 4,5 \\
49,4 \\
19,3 \\
8,6 \\
6,6 \\
\mathrm{II}, \mathrm{I} \\
7,7\end{array}$ & $\begin{array}{r}8,31 \\
2,58 \\
\text { I9, I9 } \\
3,75 \\
4,50 \\
7,73 \\
6,33 \\
1,66 \\
3,29 \\
5,75 \\
9,47 \\
9,65 \\
15,75\end{array}$ \\
\hline Moyenne $($ Mcan $)$. . . & $\begin{array}{r}19 \\
\perp \quad 14\end{array}$ & $\begin{array}{r}7,53 \\
+\quad 5,14\end{array}$ \\
\hline Canthaxanthine ro p. Ioo & hydrosoluble ( Io p. Iоo $h$ & osoluble canthaxanthin) \\
\hline $\begin{array}{l}\text { I4 } \\
\text { I5 } \\
\text { I6 } \\
\text { I } 7 \\
\text { I } 8\end{array}$ & $\begin{array}{l}43,2 \\
20,9 \\
38,6 \\
23 \\
24,4\end{array}$ & $\begin{array}{l}1,99 \\
4,08 \\
3,20 \\
1,76 \\
2,16\end{array}$ \\
\hline Moyenne (Mean) & $\begin{array}{r}30 \\
\perp \quad 10\end{array}$ & $\begin{array}{r}2,63 \\
\therefore \quad 0,97\end{array}$ \\
\hline
\end{tabular}

Le cofficient d'utilisation digestive a permis de calculer ce qui a été réellement absorbé par la truite (tabl. 3). Le rapport canthaxanthine fixée/canthaxanthine absorbée est faible : les valeurs moyennes sont égales à 7,53 pour les préparations "CARophyll rouge" et de 2,63 pour les préparations "Canthaxanthine ro p. Ioo hydrosoluble ». Les dispersions sont quant à elles de I à Io pour les préparations de "CAROPHYi, I rouge " (I,66 à I9,I9) et de I à 2 pour les préparations "Canthaxanthine Io p. Ioo hydrosoluble " (I,76 à 4,08). 


\section{Discussion}

La stabilité de la canthaxanthine, comme celle de tous les caroténoïdes, est faible, ce qui implique des précautions à prendre vis-à-vis, notamment, de son oxydation. Pour cette raison, la protection dans les concentrats est réalisée soit par enrobage du pigment par de la gélatine (présentation "CAROPHYLL rouge") soit par microdispersion dans un support glucidique (présentation Canthaxanthine Io p. Ioo hydrosoluble). Ce mode de protection semble efficace car les valeurs moyennes dont nous disposons suffisent à montrer une concordance entre le taux de canthaxanthine annoncé par le fabricant et le taux de canthaxanthine réellement trouvé dans les concentrats détenus par les fabricants d'aliments, sans précaution particulière. BUNNEI et BORENSTEIN (I967) rapportent également une bonne stabilité de la préparation "Canthaxanthine to p. Ioo hydrosoluble " et évaluent les pertes en canthaxanthine des concentrats à 0,5 p. Ioo par mois.

Dans cette expérience, les deux modes de présentation de la canthaxanthine se sont comportés de façon équivalente vis-à-vis du processus de granulation. On sait que deux facteurs, au moins, interviennent au cours de pressage : une élévation thermique et une forte pression qui entraine une abrasion lors du passage de l'aliment à travers la filière (WORNICK, I960).

L'effet thermique ne peut expliquer, à lui seul, les pertes notées car la température des granulés au sortir de la presse n'est que de $42-43^{\circ} \mathrm{C}$, températures auxquelles la canthaxanthine est stable (BORENSTEIN et BUNnEL, I966; BAUERNFEIND, I975; EMODI, Sciaipi et ANTOSHKIW, I976).

L'abrasion, davantage que la pression, serait responsable des pertes enregistrées. En effet, dans le cas de la vitamine A, également présentée sous forme enrobée, l'agglomération exerce davantage un rôle de fragilisation de l'enrobage par laminage des particules contre la filière plutôt qu'un effet de destruction proprement dit (VALDEBouze, LEVY et I,EVASSEUR, I962; VAldEBouze et LEVY, I972). Ainsi, les détériorations subies par les granulés hâteraient la destruction de la canthaxanthine par suite de la considérable surface ainsi exposée à l'air. Ceci expliquerait la dégradation également notée au cours du stockage.

La quantité de pigments caroténoïdes fixée par la truite arc-en-ciel dépend de la quantité de pigments réellement absorbée. Celle-ci ne peut s'évaluer qu'en connaissant le cœfficient d'utilisation digestive des caroténoïdes.

D'un point de vue qualitatif, aucune différence de forme dans les spectres d'absorption de la canthaxanthine, quelle que soit la préparation, n'a été observée entre la canthaxanthine ingérée et la canthaxanthine excrétée. Il semble, donc, qu'il n'y ait pas d'isomérisation de la canthaxanthine dans le tube digestif. II faut, toutefois, rester prudent car, pour le $\beta$-carotène, Mc Gil,IIVRAY (I95I) n'a pas constaté non plus d'isomérisation chez le mouton alors que ALMENDINGER et Hinds (I969) en ont observé chez le bouf.

Quantitativement, une dégradation des caroténoïdes se produit au cours du transit digestif (BooTH, 1956, I957). Si la canthaxanthine ne peut être détruite dans l'estomac, car au pH de 3, I (SchäperclaUs, I g62), elle est encore stable (BORENSTEIN et BUNNEL, I 966), elle serait dégradée dans l'intestin à pH 8 (BERNARD, I952) ou FrançoIs et LHUISSIER (I970) n'excluent pas une destruction par la flore bactérienne, d'ailleurs rapportée, chez la truite arc-en-ciel, par Trust et Sparrow (I974). Dès lors les digestibilités observées ne sont en fait que des digestibilités apparentes car la digestibilité d'un nutriment, s'obtenant par différence 
entre la quantité de ce nutriment ingéré et la quantité trouvée dans les fèces, ne tient pas compte de sa labilité.

Nos résultats font apparaître, en moyenne, une faible digestibilité de la canthaxanthine. Ils montrent, de plus, une forte variabilité entre les échantilions pour un même lot de fabrication de canthaxanthine.

Les valeurs de la digestibilité de la canthaxanthine chez la truite arc-en-ciel ne peuvent être confrontées à d'autres résultats, les données sur ce sujet étant inexistantes dans la bibliographie sur le poisson. Nous ne pouvons donc que rapprocher nos résultats de ceux obtenus chez d'autres animaux pour un pigment caroténoïde différent : le $\beta$-carotène. Chez les mammifères, on sait que le $\beta$-carotène ingéré est incomplètement absorbé (BоOTH, I956); l'utilisation digestive de ce pigment reste toutefois plus élevée que celle que nous avons notée pour la canthaxanthine; elle est de $30 \mathrm{p}$. Ioo chez le rat (BоOTH, I957) $40 \mathrm{p}$. Ioo chez la chèvre (OWEN, DARroch et Proudfoot, I 959) $40 \mathrm{p}$. IOO à $60 \mathrm{p}$. IOo chez la vache laitière (CHANDA et al., I95I). Il est important de remarquer que les pigments n'ont pas le même comportement digestif selon l'espèce animale.

Il est permis de faire des réserves sur la valeur des prélèvements de fèces effectuées par pression abdominale. En effet, si cette méthode est classiquement utilisée (SINGH et NosE, I 967). L'apparente fluctuation journalière de la composition des fèces (Possompes, I973) d'une part et la possibilité d'absorption des nutriments au niveau du rectum (Smith et Lovel, I97I; AUstreng, I978) d'autre part impliquent qu'une telle technique appliquée à un moment précis ne peut donner d'échantillonnage représentatif de la composition des fèces. Le problème est de savoir si les digestibilités mesurées à partir d'échantillons prélevés par pression abdominale conservent une signification biologique. C'est pourquoi l'élaboration d'une technique de prélèvements représentatifs de fèces de truites (CHOUBERT, De la NoüE et LuQUET, I979) permettra de préciser les résultats de ces mesures.

$\mathrm{Au}$ terme de cette expérience, toutes les truites étaient pigmentées, la différenciation, soit visuelle, soit chimique, des effets des différents lots de canthaxanthine restant impossible. En effet, si l'examen visuel des muscles de truites est aisé, il ne permet pas de définir quantitativement avec précision la valeur colorante de chacune des préparations utilisées. Fn jugeant une coloration l'œil intègre un grand nombre de données diverses dont les plus importantes sont la couleur du muscle ainsi que sa répartition sur la surface (FERRANDo et MAINGUY, I970). De plus, il ne peut être question de rattacher trop étroitement les différences de coloration observées atux dosages chimiques de la canthaxanthine. On sait en effet que l'addition d'un agent de pigmentation à la ration alimentaire peut renforcer l'impression visuelle de couleur ou la concentration du pigment dans les produits d'origine animale. Il est important de remarquer que l'un de ces effets n'implique pas nécessairement l'autre (BAUERNFEIND et al., I97r; BRUBACHER, I972).

L'examen chimique dans le muscle et le poisson entier a donné des résultats très hétérogènes quel que soit le tisstı analysé. Ils sont, toutefois, comparables à ceux rapportés par d'autres auteurs (DEUFEI, I 965; SAVOLAINEN et GYLLENBERG, I970; LAMBERTSEN et BRAEKKAN, I97I; SAITO et REGIER, I97I; AUGER, I973;

UGLETVEIT, I974; ABDUL-MALAK, I975; ABDUl-Malak et al., I975).

Les résultats très différents observés en particulier en pisciculture ne peuvent être dus à la variabilité des concentrats de canthaxanthine tels qu'ils sont incorporés dans les aliments agglomérés. Les difficultés rencontrées à cet égard dans la production de truites pigmentées proviennent de la multiplicité des paramètres, mal contrôlés, qui agissent sur la pigmentation des truites, paramètres relatifs 
à la nature dư pigment caroténoïde, à la composition de l'aliment, au poisson ou au milieu écologique (CHOUBERT, I977).

Accepté pour publication en mars 1979.

\section{Remerciements}

Nous remercions les litablissements HOFMANN-LA ROCHE ainsi que les différents fabricants d'aliments du marché français pour la fourniture d'échantillons de canthaxanthine.

\section{Summary}

Canthaxanthin content of feed concentrates as affected by pelleting and storage processes: consequences on digestibility and pigmentation of the Rainbore trout

In order to determine one of the reasons for pigmentation variability in hatchery trout, canthaxanthin losses in I 3 samples of "CAROIPYYI, red " and 5 samples of " Io p. Ioo hydrosoluble Canthaxanthin "were examined during the preservation of the feed concentrates (in beadlets) and also during the pelleting and storage processes of the feeds. The consequences of this degradation on digestibility and on pigmentation of rainbow trout were studied.

The protection of canthaxanthin in beadlets is efficient and the various batches were homogeneous; the contents of pure product were steady and corresponded to the theoretical values ( $0.5 \pm 0.5 \mathrm{ppm})$.

Though the temperature of the pellets, when they came out of the press was moderate $\left(+3^{\circ} \mathrm{C}\right)$, the pelleting process led to a large loss of pigments (table $\mathrm{I}$ ). This decrease was significant and equal to zo and i 5 p. Ioo respectively in "CAROPIIY,L, red" and " ro p. 100 hydrosoluble Can thaxanthin ".

During storage of the pellets for 2 months under ambient winter temperature, and before use, a further significant loss of about 17 p. I 00 was recorded, whatever the type of beadlets.

In vivo test showed a low apparent digestibility of canthaxanthin (20 p. 100) (table 3). These low values may be explained, at least partially, by the method used for faeces collecting.

Though the "fixed canthaxanthin/ingested canthaxanthin "ratio (table 2) was near I p. Ioo, the trout flesh was strongly coulored, irrespective of the canthaxanthin preparation used.

\section{Références bibliographiques}

ABDUL-MALAK N., I975. Influence de certains facteurs nutritionnels et écologiques sur le métabolısme d'un pigment caroténoïde : Ia canthaxanthine, chez la truite (Salmo gairdnevii R.). Thèse Doctorat $3^{\mathrm{c}}$ cycle, Lyon, ro6 p.

ABdul-Mai,ak N., ZWingeistein (a., Jouanneteau J., KoEnig J., r975. In fluence de certains facteurs nutritionnels sur la pigmentation de la truite arc-en-ciel par la canthaxanthine. Ann. Nutr. Alim., 29, 459-475.

Almendinger R., Hinds F. C., r969. Apparent carotenoid increases in the digestive tract of beef cattle. $J$. Nutr., 97, I3-18.

AUGER G., I973. La canthaxanthine: son influence sur la coloration de la chair des truites. Thèse Doct. Vété., Paris, I I 2 p.

AUSTRENG E., $197^{8}$. Digestibility determination in fish using chromic oxide marking and analysis of contents from different segments of the gastrointestinal tract. Aquaculture, 18, 265-272.

Bauernfeind J. C., I975. Carotenoids as food colors. Food technol., 29 (5), 48-49. 
Bauernfeind . C., Brubacher G. B., KLäUi H. M., Marusich W. L., I97I. Use of carotenoids in: Isler O., Cavotenoids, 743-77\%. Birkhäuser Verlag, Basel.

Bernard F., 1952. La digestion chez les poissons. Trav. Lab. Hydrobiol. Piscic. Univ., Grenoble, 44, 6I-95.

BESSE P., I95I. La saumonisation artificielle des salmonidés, truites et saumons de fontaine. C.R. Acad. Sci., 233, 637-639.

BIRD F. H., 1953. The problem of pigmentation in broilers. Poult. Dig., 12, 96-тог.

Bolin D. W., KING R. P., KLosterman E. W., I952. A simplified method for the determination of chromic oxide $\left(\mathrm{Cr}_{2} \mathrm{O}_{3}\right)$ when used as an index substance. Science, 116 (2), 634-635.

BoorH V. H., I956. Disappearance of carotene from the alimentary tract of vitamin A deficient rats. Brit. J. Nutr., 10, $24 \mathrm{I}-25^{\circ}$.

Bоотн V. H., 1957. Effect of sex on the disappearance of carotene from the alimentary tracts of rats. Brit. J. Nutr., 11, I $48-152$.

BORENSTEIN B., BUNNEL, R. H., I966. Carotenoids: properties, occurence and utilization in foods. Adv. Food Res., 15, 195-276.

BRUBACHER G. B., I972. L'importance des caroténoïdes en alimentation animale. Doc. Roche, I 3 I6, 3 I p.

BUNNEI, R. H., BoRENSTEIN B., I967. Canthaxanthin, a potential new food Color. Food Technol., 21, 33 I-334.

Chanda R., Clapham H. M., Mc Navght M. L., Owen E. C., r95r. The use of chromium sesquioxide to measure the digestibility of carotene by goats and cows. J. Agric. Sci., 41, I $79-186$.

Choubert G. (Jr), I977. Caroténö̈des fixés par la truite arc-en-ciel en croissance. Thèse Doctorat, $3^{\mathrm{e}}$ cycle, Paris, 47 p.

Choubert G. (Jr), de la NoüE J., LuQuet P., I979. Continuous quantitative automatic collector for fish feces. Prog. Fish Cult. $4 \mathrm{I}$ (2), 64-67.

Choubert G. (Jr), Luguet P., I975. Nature des caroténoïdes fixés au niveau de la peau et du muscle de la truite arc-en-ciel ayant ingéré de 1'huile rouge de Capelan. Ann. Hydrobiol., 6 (2), I $23^{-1} 3^{\circ}$.

Deufer J., I965. Pigmentierungsversuche mit Canthaxanthin bei Regenbogenforellen. $A r c h$. Fischwiss., 16, I25-132.

EDIN H., 19I8. Orienterande försök over användbarheten av en pa led-kroppsprincipen grundad metod att bestämma en foderblandings smältbarhed.

Emodi A., Scralpi L., ANToshkiw T., i976. Water dispersible optically clear carotenoid colors. Food Technol., 30, 58-60.

Ferrando R., Mainguy P., r970. La couleur du poulet de chair, compte rendu d'expérimentation. Doc. Roche, $1277,33 \mathrm{p}$.

Françots A. C., Lhuissier M., I970. L'adjonction de vitamines aux aliments de l'homme et des animaux. Ann. Nutr. Alimen., 24, 477-551.

GoodwIN T. W., I952. The comparative biochemistry of the carotenoids, 356 p., Chapman and Hall, London.

HatA M., HATA M., I973. Studies on astaxanthin formation in some fresh-water fishes. Tolhoku J. Agric. Res., 24 (4), r92-196.

Islek O., OfNER A., SIEMERS G. F., 1958. Industrial syntheses of carotenoids for use as food colors. Food Technol., 12, 520-526.

KuHN R., Sorensen N. A., I9j8. Über Astaxanthin und Ovoverdin. Ber. Dt Chem. Ges., 71, I 879 -I 888 .

LEDERER E., I935. Les caroténoüdes des animaux. Hermann et Cie, ed. Paris. Actualités Scientifiques et industrielles, 137, $60 \mathrm{p}$.

LAMBERTSEN G., BRAEKKAN O. R., I97I. Method of analysis of cathaxanthin and its occurence in some marine products. J. Sci. Fd.Agric., 22, 99-10I

MCGillivray W. A., I95I. The apparent intestinal synthesis of carotene by sheeps. Brit. J. Nutr., 5, 223-228.

Mainguy P., Rouques A., ig65. Le jaune de l'ceuf. I. F́tude générale de sa couleur. Bull. Soc. Scient. Hyg. aliment., 53 (4-5-6), 83-1 I6.

Osadca M., ARAujo M., de RITTER E., 1972. Determination of canthaxanthin in concentrates and feeds. J. Ass. off. Analyt. Chem., 55, IIo-II3

OWEN E. C., DARroch R. A., Proudfoot R., I959. Studies of the tate of passage and disappearance in the intestine of the goat of carotene dissolved in fat and mixed with chromium sesquioxide. Brit. J. Nutr., 13, 26-37. 
Peterson D. H., Jäghr H. K., Savage (;. M., Washbtik (G. N., Westeks H., ig66. Natural coloration of trout using xanthophylls. Trans. Am. Fish Soc., 95, 408-4 I4.

Possompes B. P., 1973. Infuence de la température sur les besoins en protéines, le transit alimentaire et la digestibilité chez la truite arc-en-ciel, Salmo gairdnerii Rich. Thèse Doctorat $3^{c}$ cycle, Paris, $5^{8} \mathrm{p}$.

SAIto A., REgier I. W., T97I. Pigmentation of brook trout (Salvelinus fontinalis) by feeding dried crustacean waste. J. Fish. Res. Bd Can., 28 (4), 509-512.

SAVOraINEN J. E. T., (XYlLIANBERG H. G., I970. Feeding of rainbow trouts with Rhodotorula sannëi preparations. III. Amounts and qualities of carotenoids. Iebensm. -. Wiss. u. Technol., 3, I $8-20$.

SCHÄPERCIAUs W., I962. Traité de pisciculture en étang, 620 p., Vigot lìrères éd. Paris.

Shnarfich I. D., Sakinfinko E. G., I97I. Dynamics of carotenoids in tissus and organs of fish relative to the sexual cycle (en russe). Gidrobiol. Zh., 7 (6), 90-93.

SiNGH R. P., Nose, T., rg67. Digestibility of carbohydrates in young rainbow trout. Bull. Feshwater Fish Res. Lab., 17 (I), $2 \mathrm{I}-25$.

Slinger s. J., Razzaduf A., Cho C. Y., 1978. Effect of feed processing and leaching on the losses of certain vitamins in fish diets. EIFAC/78/SYMP.E/7o, i 8 p.

Smith B. W., LOVFi, R. T., I97I. Digestibility of nutrients in semi-purified rations by cliannel catfish in stainless steel troughs. Proc. Annu. Conf. Southeast. Asso. Game Irish. Comm., 25, $452-459$.

STEVEN D. M., I948. Studies on animal carotenoids: I. Carotenoids of the brown trout (Salmo trutter Linn). J. Exp. Biol, 25, 369-387.

STIVEN D. M., I949. Studies on animal carotenoids. II. Carotenoids in the reproductive cycle of the brown trout. J. Exp. Biol., 26, 295-303.

Sumper I. B., Fox D. L., I933. A study of variations in the amount of yellow pigment (xanthopliyll) in certain fishes and the possible effects upon this of colored backgrounds. J. Exp. Zool., 66 (2), 263-3or

Trust T'. J., SPARRow R. A. H., I974. The bacterial flora in the alimentary tract of freshwater salmonid fishes. Can. J. Mucrobiol., 20, 12 I9-1228.

Ugletviit S., I974. Pigmentering av lakse-og ørretkjøtt. Fisken Hav. ser. B., 9, 3I-6o.

VALDFBOUze P., LEVy B. R., I972. Influence de l'agglomération et du stockage des aliments composés sur leur teneur en vitamine A. Ind. Alim. Anim., 2, 49-54.

VAldibouze P., Levy B. R., Levasseur L., i 962 . La stabilité de la vitamine A dans les produits destinés à l'alimentation des animaux. Ind. Alim. Anim., 134, 7r-77.

WORNICK R. C., I960. L'agglomération des aliments composés et ses effets sur les substances auxıliaires. Ind. Alim. Anim, 105, $3 \mathrm{I}-48$.

Yvoris P., MAINGUY P., i972. Incidence des coccidioses sur le métabolisme des caroténoïdes. Essai d'interprétation pathogénique. Doc. Roche, I302, $50 \mathrm{p}$. 